\title{
Longitudinal Stability Calculations
}

\author{
M. Blaskiewicz
}

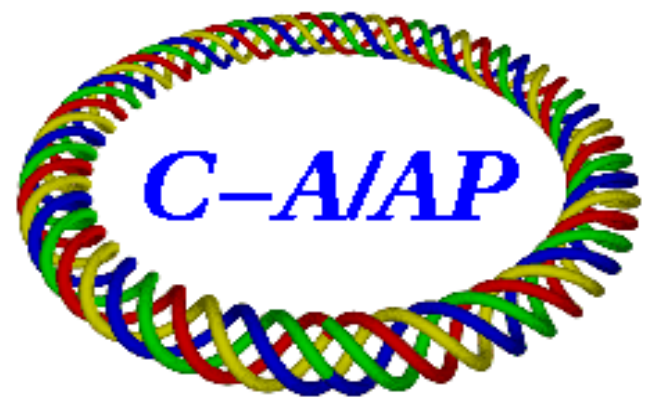

\section{Collider-Accelerator Department Brookhaven National Laboratory Upton, NY 11973}

Notice: This document has been authorized by employees of Brookhaven Science Associates, LLC under Contract No. DE-AC02-98CH10886 with the U.S. Department of Energy. The United States Government retains a nonexclusive, paid-up, irrevocable, world-wide license to publish or reproduce the published form of this document, or allow others to do so, for United States Government purposes. 


\title{
Longitudinal Stability Calculations
}

\author{
M. Blaskiewicz* \\ BNL, Upton NY 11973, USA
}

\begin{abstract}
Coupled bunch longitudinal stability in the presence of high frequency impedances is considered. A frequency domain technique is developed and compared with simulations. The frequency domain techniqe allows for absolute stability tests and is applied to the problem of longitudinal stability in RHIC with the new $56 \mathrm{MHz}$ RF system.
\end{abstract}

*Electronic address: blaskiewicz@bnl.gov 


\section{THEORY}

The problem of bunched beam longitudinal stability has been discussed many times [19]. In the present treatment it is shown that one can extend dispersion integral techniques to longitudinal modes with complicated internal bunch structure. We assume $M$ identical equally spaced bunches. Let $\theta$ denote the azimuth, which increases by $2 \pi$ each turn, $T_{0}$ be the synchronous revolution period and $\omega_{0}=2 \pi / T_{0}$ be the angular revolution frequency. Take $\phi=\theta-\omega_{0} t$ as the longitudinal coordinate and consider a driving voltage $\hat{V} \exp \left(i\left(k_{0} M+\right.\right.$ $s) \phi-i \Omega t$ ) where $s$ is the coupled bunch mode number and $\Omega$ is the fractional drive frequency. In amplitude angle coordinates $\phi=r \sin \psi$ and the Vlasov equation reads

$$
-i \Omega F_{1}+\omega_{s}(r) \frac{\partial F_{1}}{\partial \psi}=\frac{\partial H_{1}}{\partial \psi} \frac{d F_{0}}{d r}
$$

where $F_{0}(r)+F_{1}(r, \psi) \exp (-i \Omega t)$ is the normalized distribution function for the first bunch, $\int F_{0}(r) 2 \pi r d r=1$. The perturbation hamiltonian is due to the applied voltage and the beam induced voltage

$$
H_{1}=\frac{\bar{\eta} q \omega_{0}}{2 \pi r \omega_{s 0}}\left\{\frac{\hat{V} e^{i\left(k_{0} M+s\right) r \sin \psi}}{i\left(k_{0} M+s\right)}-\sum_{k \neq 0} \frac{\rho_{k} Z_{k}}{i(k M+s)} e^{i(k M+s) r \sin \psi}\right\}
$$

where $\bar{\eta}=\omega_{0} \eta /\left(\beta^{2} E_{0}\right), q$ is the charge per particle, $\omega_{s 0}$ is the small amplitude synchrotron frequency, $Z_{k}=Z\left[\omega_{0}(k M+s)+\Omega\right]$, and

$$
\rho_{k}=\frac{q \omega_{0} N M}{2 \pi} \int r d r d \psi F_{1}(r, \psi) e^{-i(k M+s) r \sin \psi}
$$

where there are $N$ particles per bunch.

To solve the system take

$$
F_{1}(r, \psi)=\sum_{\ell \neq 0} R_{\ell}(r) e^{i \ell \psi}
$$

where $\ell$ is the synchrotron mode number. Insert eq (4) into eq (1), multiply by $\exp (-i m \psi) d \psi / 2 \pi$ and integrate over $\psi$,

$$
\begin{aligned}
{\left[-i \Omega+i m \omega_{s}(r)\right] R_{m}(r) } & =\oint \frac{d \psi}{2 \pi} e^{-i m \psi} \frac{\partial H_{1}}{\partial \psi} \frac{d F_{0}}{d r} \\
& =i m F_{0}^{\prime} \oint \frac{d \psi}{2 \pi} e^{-i m \psi} H_{1}(r, \psi)
\end{aligned}
$$




$$
=F_{0}^{\prime} \frac{\bar{\eta} q m \omega_{0}}{2 \pi r \omega_{s 0}}\left\{\frac{\hat{V} J_{m}\left[\left(k_{0} M+s\right) r\right]}{k_{0} M+s}-\sum_{k \neq 0} \frac{\rho_{k} Z_{k}}{k M+s} J_{m}[(k M+s) r]\right\}
$$

Now define

$$
C_{m, p}=\int_{0}^{\hat{\phi}} r d r R_{m}(r) J_{m}[(p M+s) r]
$$

so that

$$
\rho_{k}=q \omega_{0} N M \sum_{\ell \neq 0} C_{\ell, k}
$$

Inserting this is eq (5) gives

$$
C_{m, p}=\frac{\bar{\eta} q^{2} \omega_{0}^{2} M N}{2 \pi \omega_{s 0}} \sum_{k} \frac{Z_{k}}{k M+s} m \int_{0}^{\hat{\phi}} \frac{d r J_{m}[(p M+s) r] J_{m}[(k M+s) r]}{-i \Omega+i m \omega_{s}(r)} F_{0}^{\prime}(r) \sum_{\ell \neq 0} C_{\ell, k}+d r i v e .
$$

Summing over $m$ gives

$$
\rho_{p}-\frac{\bar{\eta} q^{2} \omega_{0}^{2} M N}{2 \pi \omega_{s 0}} \sum_{k} \frac{Z_{k}}{k M+s} \rho_{k} \sum_{m} m \int_{0}^{\hat{\phi}} \frac{d r J_{m}[(p M+s) r] J_{m}[(k M+s) r]}{-i \Omega+i m \omega_{s}(r)} F_{0}^{\prime}(r)=\text { drive. }
$$

The integral in equation (7) can be singular if $\operatorname{Im}(\Omega)=0$. For this case I consider $\Omega=\operatorname{Re}(\Omega)+i 0^{+}$, a small positive imaginary component corresponds to an adiabatic turn on of the driving force. Equation (7) is also valid for finite growth rates with $\operatorname{Im}(\Omega)>0$. For $\operatorname{Im}(\Omega)<0$, equation $(7)$ is not trustworthy. In particular, a large negative imaginary part for the drive will decay faster that the most rapidly damping coherent mode. Clearly, the beam response will decay like the coherent mode, not the drive. Other subtleties, like the infinite drive size in the far past, also need to be carefully considered.

Even with the restriction $\operatorname{Im}(\Omega)=0^{+}$it is possible that eq $(7)$ will not have a solution. This will be the case when the system is unstable and this is the key to using it for predicting beam stability. In particular notice that (7) is of the form

$$
[\mathbf{1}-N \mathbf{Z}(\Omega)] \mathbf{P}=\mathbf{D}
$$

where $N$ is the number of particles per bunch, $\mathbf{P}=\rho_{p}, \mathbf{Z}$ is the rest of the dispersion matrix, and $\mathbf{D}$ is the drive. For small $N, \mathbf{P} \approx \mathbf{D}$ and the system is stable. As $N$ grows the matrix $\mathbf{1}-N \mathbf{Z}(\Omega)$ changes until, for some $\Omega=\Omega_{c}, \operatorname{det}\left(\mathbf{1}-N_{t} \mathbf{Z}\left(\Omega_{c}\right)\right)=0$, where $N_{t}$ is the threshold intensity for coherent frequency $\Omega_{c}$. Therefore, to use (7) in a stability analysis one plots 
$\operatorname{det}(\mathbf{1}-N \mathbf{Z}(\Omega)])$ on the complex plane as a function of $\Omega$. Strictly speaking these plots should also be made for all values of $N$ less than the value of interest. If none of the plots encircle the origin the matrix will have an inverse for the intensity of interest and the system will be stable. If the curve goes through the origin then the frequency $\Omega$ is an eigenfrequency.

To connect this formalism to the usual results note that retaining a single value of $m$ in the summation of $(7)$ and making the approximation $J_{m}(x)=(x / 2)^{m} / m$ ! leads to a matrix of rank one. The resulting coherent frequencies are similar to the handbook formulas [6].

\section{APPLICATIONS}

The theory in the previous section has been implemented in the fortran code NYQUIST. NYQUIST does not address the $s=0$ mode since the phase, radial and RF feedback loops have not been included. I assume the frequency shifts will be small so that only one synchrotron mode at a time needs to be considered. That is to say, the sum over $m$ in (7) is replaced by a single value. The variation of synchrotron frequency with $r$ is taken as $\omega_{s}(r)=\omega_{s 0}(h r / 4)^{2}$ where $h$ is the harmonic number. The infinite sum over $k$ in (7) is truncated according to $\left|f_{0}(k M+s)\right|<f_{\max }$. The impedance is modeled as a sum of resonators plus a constant, broad band $Z / n$. The dispersion integrals are treated numerically using a uniform grid in the action-like variable $I=(h r)^{2}$. For $\operatorname{Im}(\Omega)=0$ one takes

$$
\frac{1}{0^{+}-i \Omega+i m \omega_{s 0} I / 16}=i P V\left\{\frac{1}{\Omega-m \omega_{s 0} I / 16}\right\}+\pi \delta\left(\Omega-m \omega_{s 0} I / 16\right) .
$$

By using an action grid $I_{n}=n \Delta I$ and a frequency grid $\Omega_{k}=k \Delta I m \omega_{s 0} / 16$, the delta function always corresponds to a lattice point and the principle value is just a sum with the single point corresponding to the resonant denominator removed. For $\operatorname{Im}(\Omega)>0$ we take $\Omega_{k}=i \epsilon+k \Delta I m \omega_{s 0} / 16$ to keep as much symmetry as possible. Also we take $\Delta I m \omega_{s 0} / 16 \lesssim \epsilon / 5$ which corresponds to a $1.3 \%$ fractional error between summation and integration. The fractional error was estimated using (for positive $a$ )

$$
\sum_{k=-\infty}^{\infty} \frac{a}{a^{2}+k^{2}}=\sum_{k=-\infty}^{\infty} \pi e^{-a|k|}=\pi+\frac{2 \pi}{e^{a}-1}
$$

The accuracy and convergence of NYQUIST has been benchmarked using simulations. Figure 1 shows the result of simulations of four bunches with 20,000 macroparticles each. 


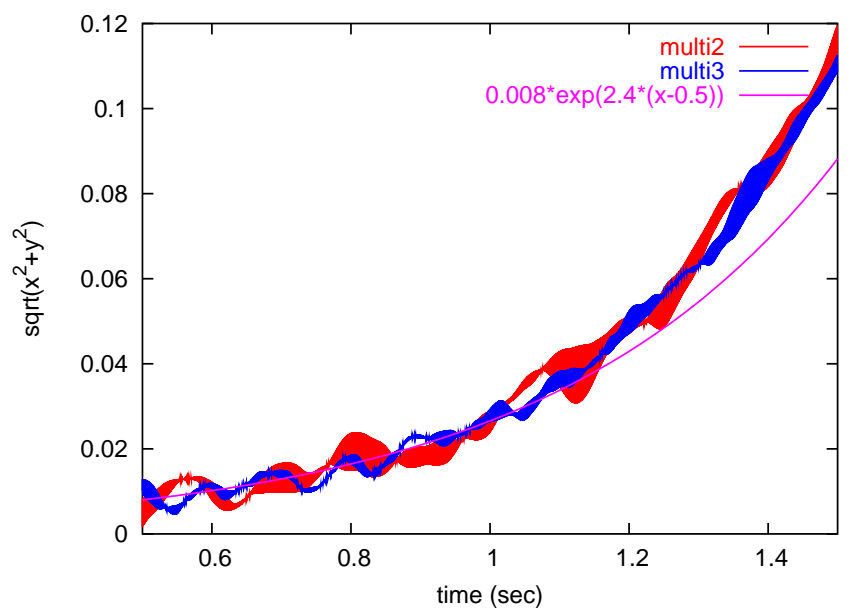

FIG. 1: Comparison of multiparticle simulations and the threshold of NYQUIST.

The impedance consists of a broad band $Z / n$ and a narrow band resonator. The third revolution hamonic of the beam current is plotted as a function of time. For the red curve labeled multi2 the intital phase space distribution of macroparticles was regular and the charge per macroparticle was adjusted to obtain the desired line density. For the blue curve labeled multi3 all macroparticles had the same charge and the initial phase space distribution was chosen to give the correct line density. The two curves agree rather well. The magenta curve shows an exponential with a $2.4 \mathrm{~s}^{-1}$ growth rate.

Figure 2 shows results from NYQUIST with a $2.4 \mathrm{~s}^{-1}$ growth rate for different values of $f_{\text {max }}$. All come close to the black dot at the origin but the zoomed in version of Figure 3 shows that the agreement is not perfect. The equivalent length of the smoothing function was $\tau_{s}=5 \mathrm{~ns}$. The corresponding upper frequency is $f_{\max }=1 / 2 \tau_{s}=100 \mathrm{MHz}$ so the the black curve is closest to the simulation. Figure 4 shows NYQUIST results near the origin for $f_{\max }=100 \mathrm{MHz}$ and a growth rate of $1.2 \mathrm{~s}^{-1}$ if found. This is a factor of 2 smaller than the simulation and it is possible that the smooth spectral cutoff used in the simulation behaves differently than truncating the matrix. Also note that the linear $\mathrm{rf}$ growth rate for this system is $4.8 \mathrm{~s}^{-1}$ so the effects of Landau damping are large.

The code has been checked for internal consistency and there appear to be no mistakes. In particlular, the long wavelength limit has been checked and agrees quite well with the usual formulas $[3,4]$. Therefore, I will make the assumption that the calculations are reliable.

First I ignore the broad band impedance. Also I ignore coupling between the rings. Figure 5 shows nyquist curves for a bright beam and compares the $308 \mathrm{MHz}$ higher order 


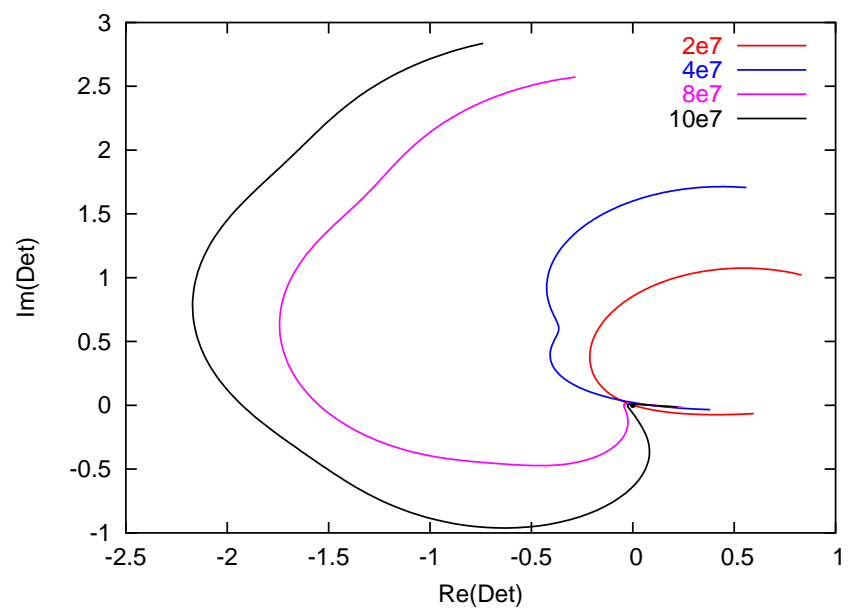

FIG. 2: NYQUIST threshold curves versus upper frequency cutoff. $\operatorname{Im}(\Omega)=2.4 \mathrm{~s}^{-1}$

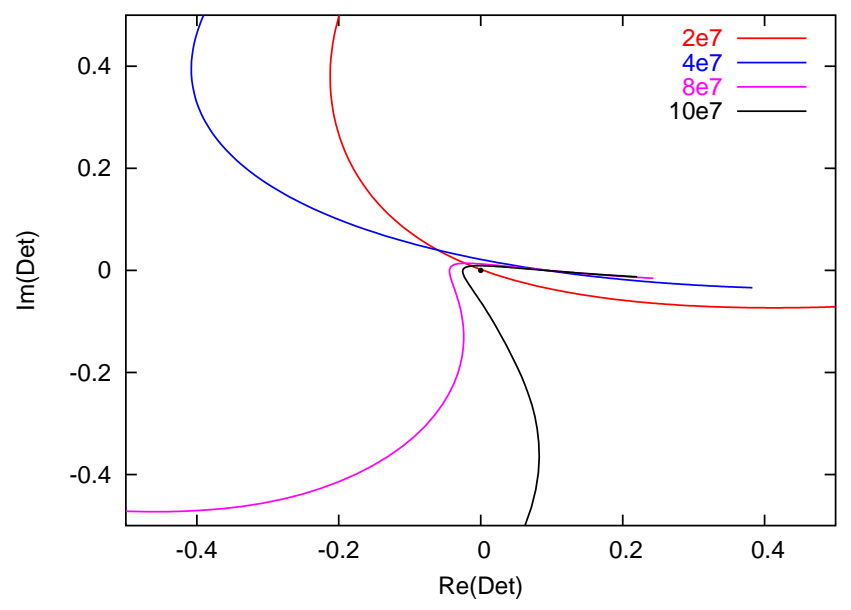

FIG. 3: Zoomed in version of Figure 2.

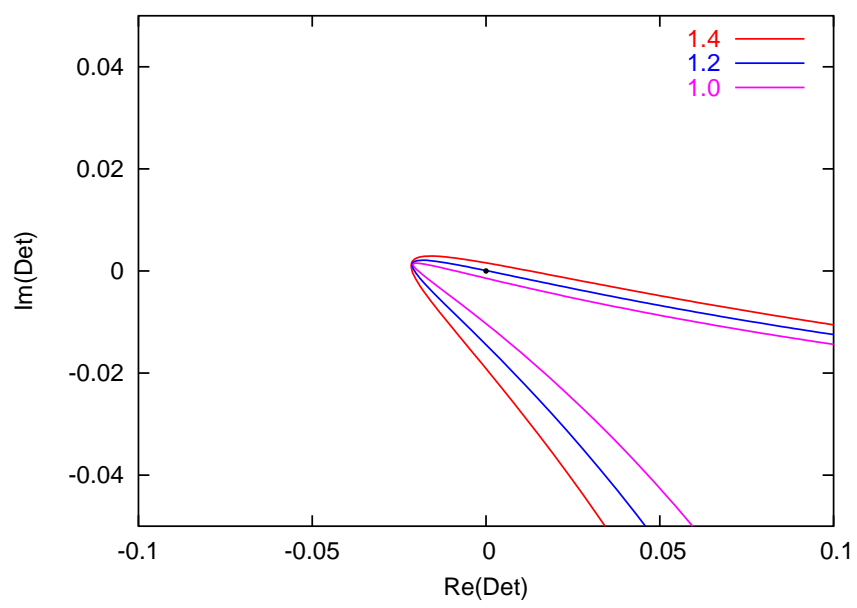

FIG. 4: Curves for $f_{\max }=10^{8} \mathrm{~s}^{-1}$ and different growth rates. 


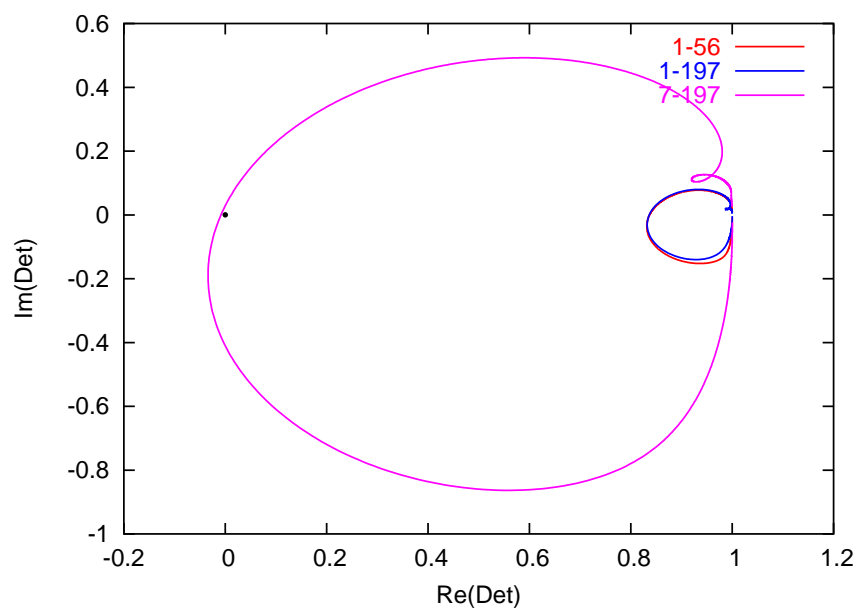

FIG. 5: Plot of the determinant for $\operatorname{Im}(\Omega)=1 \mathrm{~s}^{-1}$ and $2 \times 10^{11}$ protons with $2.4 \mathrm{MV}$ on $56 \mathrm{MHz}$ cavity. The $m=1$ dipole mode is plotted. There were 720 bunches in the calculation but the narrow band $\mathrm{Z}$ was reduced by a factor of 6 . Broad band $\mathrm{Z}$ unchanged.

mode (HOM) on the $197 \mathrm{~s}$ with the $168 \mathrm{MHz} \mathrm{HOM}$ on the $56 \mathrm{MHz}$ cavity. Measurements of frequency differences between the various 197s suggest that each cavity will contribute to a different coupled bunch mode so the blue and red curves should be compared. For this bunch length and no broad band $Z / n$ the 197 s and 56 are pretty close and neither cause a $1 \mathrm{~s}^{-1}$ grow rate.

Figure 6 includes the broad band impedance with a growth rate of $0.1 \mathrm{~s}^{-1}$. Calculations wer done for root mean square (rms) bunch lengths of $1.6 \mathrm{~ns}$ and $2.2 \mathrm{~ns}$. Figure 7 shows what happens in the limit $\operatorname{Im}(\Omega) \rightarrow 0$. Stability for the $1.6 \mathrm{~ns} \mathrm{rms}$ length is marginal but things are very stable for $2.2 \mathrm{~ns}$.

The calculations in Figures 5,6, and 7 only included the lowest frequency HOMs in Table II. To get an idea of a worst case scenario all the $56 \mathrm{MHz}$ modes with a resonant frequency less than $600 \mathrm{MHz}$ where shifted in frequency to drive the same CBM.

\section{CONCLUSIONS}

Longitudinal coupled bunch instabilities due to HOMs in the $56 \mathrm{MHz}$ cavity have been considered. The addition of the $56 \mathrm{MHz}$ impedance does not have a signficant impact on 


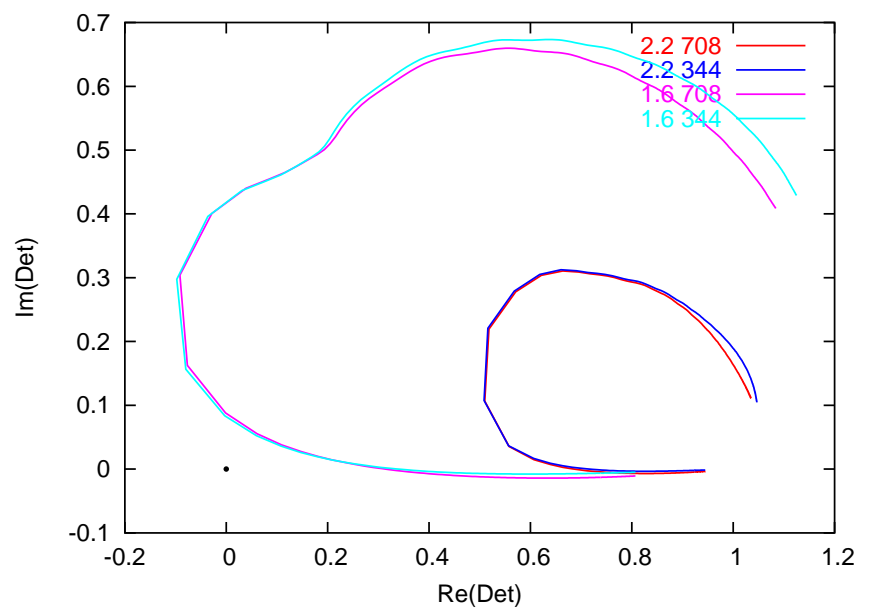

FIG. 6: Plot of the determinant for $\operatorname{Im}(\Omega)=0.1 \mathrm{~s}^{-1}$ and $1 \times 10^{11}$ protons with $2.4 \mathrm{MV}$ on the $56 \mathrm{MHz}$ cavity , mode 344 is for the $197 \mathrm{MHz}$ HOM mode 708 is for the $56 \mathrm{MHz}$ cavity. There were 720 bunches in the calculation so the narrow band $Z$ was reduced by a factor of 6 . The broad band $Z$ was unchanged.

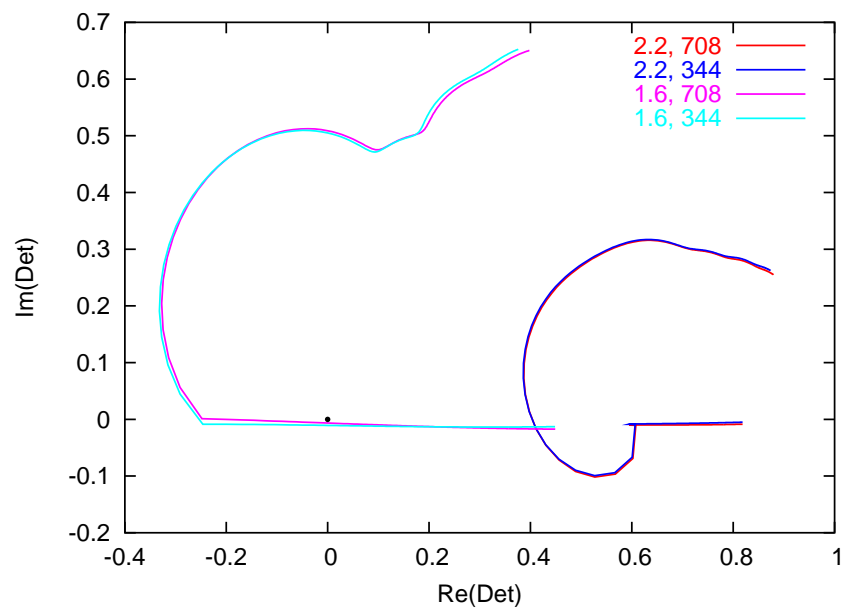

FIG. 7: Plot of the determinant for $\operatorname{Im}(\Omega)=0$ and $1 \times 10^{11}$ protons with $2.4 \mathrm{MV}$ on the $56 \mathrm{MHz}$ cavity. Bunch lengths with $\sigma_{t}=1.6$ ns and $2.2 \mathrm{~ns}$ are shown. Other details are like in Figs 5 and 6

the threshold of longitudinal instabilities.

[1] M.Q. Barton and E.C. Raka, PAC71 p1032 (1971).

[2] F. Sacherer PAC73 p825 (1973).

[3] J.M. Wang BNL 51302 (1980). 


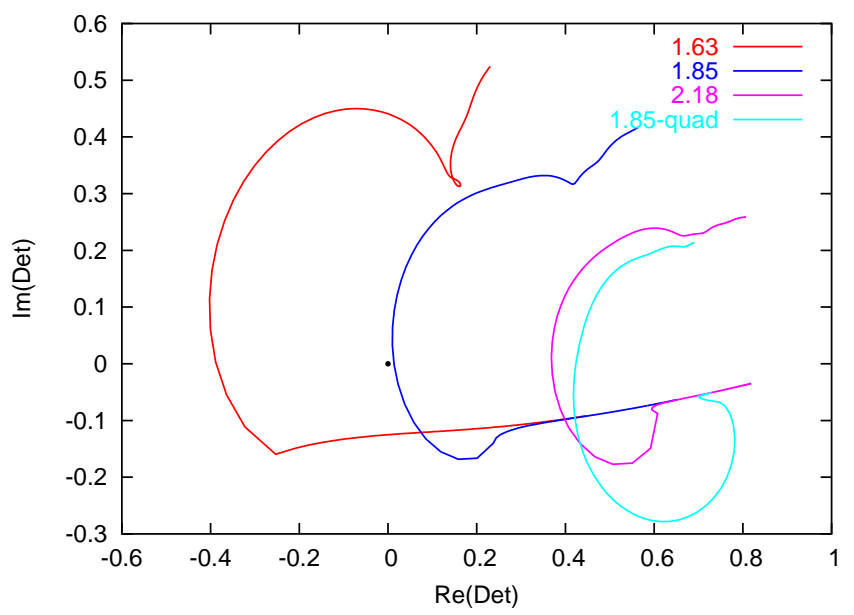

FIG. 8: Plot of the determinant for $\operatorname{Im}(\Omega)=0$ and $1 \times 10^{11}$ protons with $2.4 \mathrm{MV}$ on the $56 \mathrm{MHz}$ cavity. Bunch lengths with $\sigma_{t}=1.6 \mathrm{~ns}, 1.8 \mathrm{~ns}$ and $2.2 \mathrm{~ns}$ are shown. Also, the quadrupole $(m=2)$ mode for the $1.8 \mathrm{~ns}$ mode is shown. All the $56 \mathrm{MHz}$ modes below $600 \mathrm{MHz}$ where adjusted slightly in frequency to drive the same, 708, CBM. There were 720 bunches used for the calculation and the narrow band impedance was reduced by a factor of 6 to simulate 120 bunches.

[4] B. Zotter CERN SPS/81-19 (DI) (1981).

[5] Y.H. Chin, K. Satoh, K. Yokoya, Particle Accelerators 13 p 45 (1983).

[6] S. Krinsky, J.M. Wang, Particle Accelerators 17 p 109 (1985).

[7] J.L. Laclare, CERN 87-03, p264 (1987).

[8] R. Baartman, S. Koscielniak, Particle Accelerators 28 p 95, (1990).

[9] S.Y. Zhang, W. T. Weng, Workshop on Beam Instabilities in Storage Rings, Hefei, China July

\begin{tabular}{|c|c|}
\hline parameter & value \\
\hline $\mathrm{h}=360$ voltage & $300 \mathrm{kV}$ \\
$\mathrm{h}=720$ voltage & $2.4 \mathrm{MV}$ \\
FWHM bunch length & $10 \mathrm{~ns}$ to $3 \mathrm{~ns}$ \\
particles/bunch & $10^{11}$ \\
\hline Lorentz factor & 107 \\
circumference & $3834 \mathrm{~m}$ \\
transition gamma & 22.89 \\
\hline
\end{tabular}

TABLE I: Machine and Beam Parameters 
1994; BNL-60749 (1994).

\begin{tabular}{|c|c|c|}
\hline$f_{r}(\mathrm{MHz})$ & $R / Q(\mathrm{Ohm})$ & $Q$ \\
\hline $56 \mathrm{MHz}$ & & \\
56.2 & 46.1 & 14760 \\
168.0 & 11.4 & 3840 \\
384.0 & 9.4 & 7440 \\
485.2 & 27.1 & 13090 \\
584.9 & 21.1 & 8560 \\
688.1 & 7.5 & 630 \\
795.6 & 1.5 & 2670 \\
1105 & 29.4 & 5650 \\
\hline $197 \mathrm{MHz}$ & & \\
197.1 & 170 & 49260 \\
308.4 & 12 & 4400 \\
445.0 & 7 & 1200 \\
543.1 & 43 & 690 \\
604.5 & 13 & 1500 \\
844.5 & 1 & 1000 \\
993.4 & 4.6 & 9900 \\
\hline
\end{tabular}

TABLE II: Higher order modes (HOMs) for the $56 \mathrm{MHz}$ cavity and one $197 \mathrm{MHz}$ cavity. 